\title{
Fertility preservation in pediatric and young adult female cancer patients
}

\author{
Hashin Kim, MD, \\ Hoon Kim, MD, PhD, \\ Seung-Yup Ku, MD, PhD, \\ Department of Obstetrics and \\ Gynecology, Seoul National \\ University College of Medicine, \\ Seoul, Korea
}

As the 5-year survival rate increases up to $80 \%$ in pediatric cancer patients, the number of women patients with reduced gonadal function by chemotherapy and radiotherapy increases. The gonadal toxicity of pediatric patients varies highly according to the chemotherapeutic agent and the type of radiotherapy. Although American Society of Clinical Oncology published the guideline for fertility preservation, additional scientific and ethical concerns should be considered for clinical practice. In addition, only the experimental method can be applied for the prepubertal patients in contrast to the postpubertal patients. In this review, we will discuss some options for preserving fertility among women's quality of life issues.

Keywords: Fertility preservation, Pediatric cancer, Female infertility

\section{Introduction}

Childhood cancer is a rare disease and the prevalence is researched to be approximately 17.7 per 100,000 children in the United States in 2012. ${ }^{1)}$ In Korea, about 1,700 children and adolescents (aged 0-19 years) were diagnosed as a maligancy and the incidence is estimated to be approximately 16 per 100,000 in 2015 . $^{2}$ For the last 40 years, childhood cancer survival rates have been steadily increasing and data suggests that over $80 \%$ of young people diagnosed as cancer will survive over 5 years and $70 \%$ will be long-term cancer survivors with recent advancement in the diagnosis and treatment. ${ }^{3)}$ Trends in 10-year relative survival of patients with major childhood cancer, aged 0-17 years, have continued to be improved and 10year relative survival rate in Korea exceeded 75\% in 2006-2010. ${ }^{2)}$ With the improvement of prognosis, it is time to consider quality of life following treatment and now childbearing and fertility preservation have become a momentous issue for cancer survivors and their parents.

Fertility preservation should be consulted with the patients and their parents before beginning treatments. Expert groups including the American Society of Clinical Oncology and the American Society for Reproductive Medicine stressed the need to discuss accessible options for fertility preservation or refer patients to the specialists in reproductive

Received: 14 June, 2018

Accepted: 14 June, 2018

Address for correspondence:

Seung-Yup Ku, MD, PhD

Department of Obstetrics and Gynecology, Seoul National University College of Medicine, 101, Daehak-ro, Jongno-gu, Seoul 03080, Korea

Tel: +82-2-2072-2387

Fax: +82-2-762-3599

E-mail: jyhsyk@snu.ac.kr

https://orcid.org/0000-0002-6423$854 \mathrm{X}$ endocrinology to make the process for decision easier. ${ }^{4,5)}$ Although the significance of fertility issues and counseling with the oncologist is more emphasized, many patients get little information from primary oncologists and the doctors feel uncomfortable to offer sufficient counseling to patients and their parents. ${ }^{6}$ In a recent study, less than $50 \%$ of oncologist referred their patients to reproductive specialists to discuss fertility risks in the United States and Canada. $^{7,8)}$

This article reviews the effects of treatment of female pediatric and adolescent cancer on infertility and the treatment options for preserving fertility. 


\section{Why is early referral to specialists in fertility preservation important for patient?}

Several international guidelines has already recommended early referral of young patients diagnosed with cancer to reproductive endocrinologists as soon as possible after diagnosis and it would decrease time spent making decisions and undergoing procedures. ${ }^{4,5)}$ Early counseling can provide a positive psychological effect for the patient and her parents, and can be accepted even when realistic fertility options are not available. Furthermore, when premature ovarian failure occurs after treatment, it may not miss giving important information such as the necessity of hormone replacement therapy and the option of oocyte donation.

In a study which investigated the importance of early referral, early referred breast cancer patients especially before breast surgery could afford more time for fertility preservation and could preserve more oocytes. ${ }^{10)}$

Early referral can also provide enough time for decisionmaking. Recently, Kim and Mersereau ${ }^{11)}$ have investigated decisional conflict scale (DCS) of the patients and fertility preservation according to the referral time. In their study, early referral (within 2 weeks of diagnosis) group was more likely to have low DCS compared to the late referral group (more than 2 weeks after diagnosis). Since it is not easy to make a decision for the patients and their children encountered a lifethreatening disease, patients should be given appropriate time to make a decision, and to make this possible, early referral to the appropriate reproductive specialists is an indispensable step.

\section{Effect of cancer treatments on fertility}

Fertility rates after cancer treatment vary with primary diagnosis and are related to the therapeutic toxicity to reproductive system. Pediatric and adolescent cancer treatment may include surgery, chemotherapy (e.g., alkylating agents), radiotherapy (RT) and/or bone marrow transplantation. Adverse events associated with cancer treatment can occur through the ovaries, or the uterus as a result of treatment.

\section{Effect of chemotherapy on the ovaries}

The ovaries are susceptible to cancer treatment because there are germ cells that cannot be regenerated from birth (nearly one million primordial follicles). Ovarian damage caused by treatment can lead to amenorrhea during or immediately after treatment and it could be temporary or permanent. Women who maintain certain degree of ovarian function after treatment is complete or who recover regular menstruation from amenorrhea may still experience problems later when trying to conceive or go through early menopause. Furthermore, regular menstruation cannot be concluded to have no damage to the ovarian reserve, so ovarian function should be judged not only by menstruation but also by anti-Mullerian hormone, follicle stimulating hormone (FSH), and ultrasound. Acute ovarian failure develops in a small subset ranged from $6 \%$ to $12 \%$ in childhood cancer survivors. ${ }^{12)}$

Although the pathophysiological mechanism of ovarian failure induced by chemotherapy is not fully understood, chemotherapeutic agents, especially alkylating agents, induce cross-linkages and destroy single stranded DNA, leading to significant DNA damage. When tissue samples are taken and histologically examined in patients who received chemotherapy, interstitial fibrosis and ovarian vascular damage were observed, as well as decreased number or complete loss of follicles. ${ }^{13)}$ The most detrimental effects were observed in alkylating agents including chlorambucil, cyclophosphamide, ifosfamide, melphalan, busulfan, and procarbazine. ${ }^{14,15)}$ Furthermore, myeoblastive chemotherapy regimens such as high-dose cyclophosphamide with busulfan or Thiotepa-based high-dose therapy for stem cell transplantation has been investigated to increase the incidence of ovarian failure (Table 1). ${ }^{16)}$

Older women are more vulnerable to same amount of chemotherapy and/or RT, compared to younger women because ovarian reserve decreases with age. In addition, the beginning of puberty is another modifier of ovarian function after treatment. In the childhood cancer survivor study, secondary amenorrhea rate of postpubertal girls was higher than the primary amenorrhea rate of prepubertal girls. Adolescents have about 2 to 3 times higher risk of premature ovarian insufficiency than those under 12 years of age. ${ }^{12)}$

Table 1. Risk of ovarian toxicity of chemotherapeutic agents

Risk of ovarian toxicity
Cyclophosphamide
Chlorambucil
Melphalan
Busulfan
Procarbazine
Dacarbazine
Ifosfamide
Chlormethine
Moderate
Cisplatin
Carboplatin
Doxorubicin (=adriamycin)
Low
Methotrexate
5-Fluoruracil
Vincristine
Bleomycin
Actinomycin D
Vinblastine
Mercaptopurine




\section{Effect of RT on the uterus and ovaries}

Radiotherapy can be used in combination with chemotherapy or used alone in pediatric cancer treatment. Human oocyte is sensitive to the harmful effects of radiation and it is generally known that a dose of 2 Gy impairs $50 \%$ of ovarian reserve function. ${ }^{17)}$ Pelvic RT can destruct ovarian follicular pool, ultimately leading to premature ovarian insufficiency. Much like chemotherapy, the risk of ovarian damage from radiation is affected by radiation field, radiation dosage, and age at the time of exposure. It can also be damaged by scattered radiation despite ovarian shielding. ${ }^{18)}$ The estimated sterilizing irradiation dose, which causes premature ovarian failure, is $20.3 \mathrm{~Gy}$ at birth, 16.5 Gy at the age of 20 years, and 14.3 Gy at the age of 40 years. ${ }^{19,20)}$. In addition, pelvic RT might cause uterine fibrosis and interrupt uterine blood flow. ${ }^{21)}$

\section{Fertility preservation options}

Young woman in reproductive age who wants to preserve fertility may choose oocyte or embryo cryopreservation. However, application of these techniques to postpubertal adolescents seems to be an additional challenge because transvaginal instrumentation with damage of hymen is needed for oocyte or embryo cryopreservation. The alternatives to oocyte cryopreservation (OC) involve ovarian tissue cryopreservation (OTC) or suppression of the effects of anticancer drugs on the ovaries through ovarian suppression (Table 2). ${ }^{22,23)}$

\section{Oocyte cryopreservation}

After 30 years of clinical practice, in vitro fertilization has now become a standardized procedure and embryo cryopreservation is one of the most well-established technique of fertility preservation. The ovaries are hyperstimulated with gonadotropic hormones to induce multiple follicle growth in the ovaries. To preserve surplus embryos, embryo cryopreservation has been also established as a common method. For postpubertal girls without a male partner, OC is another option for fertility preservation. ${ }^{9)}$ The protocol for medical ovarian stimulation via multiple hormonal injections and transvaginal oocyte retrieval in OC is quite similar to that

Table 2. Different female fertility preservation options and their feasibility to prepubertal girls

\begin{tabular}{lcc}
\hline Status & Fertility preservation options & $\begin{array}{c}\text { Feasibility to } \\
\text { prepubertal girls }\end{array}$ \\
\hline Established options & $\begin{array}{c}\text { Embryo cryopreservation } \\
\text { Oocyte cryopreservation }\end{array}$ & Not feasible \\
$\begin{array}{l}\text { Experimental options } \\
\text { Ovarian tissue cryopreservation } \\
\text { and autotransplantation } \\
\text { In vitro Maturation }\end{array}$ & Feasible \\
Debatable options & Ovarian suppression & May be feasible \\
\hline
\end{tabular}

of embryo cryopreservation. Its eventual use will require in vitro fertilization and clinical pregnancy rates (CPRs) range from $4 \%$ to $12 \%$, with $36 \%$ to $61 \%$ of CPRs per subsequent transfer of frozen embryo. ${ }^{24)}$ As such, recent studies have demonstrated that transfer of embryos originated from frozen-thawed oocytes had comparable pregnancy rates compared to those using fresh oocytes. ${ }^{25-27)}$ As freezing and thawing techniques have been improved currently, $\mathrm{OC}$ is no longer considered as an experimental method.

Even if improved technique of $\mathrm{OC}$ has resulted in more than 900 births in last 30 years, ${ }^{28)}$ there are some limitations to establish OC as a standard method for childhood and adolescent cancer patients. Firstly, OC requires time for ovarian stimulation and follicular growing, which can be especially a problem in pediatric cancers, which often require urgency to start cancer treatment. Random-start controlled ovarian stimulation protocol can result in shortening the time from consultation to cryopreservation, but even a 2 -week delay is often not feasible in cancer treatment because of characteristics of childhood cancer with aggressive prognosis. Secondly, oocytes are retrieved through transvaginal approach, which is accompanied by damage such as hymen rupture. Pediatric and adolescent patients who have never had sexual relationship or vaginal procedure previously, even if they were sexually mature, may find this processes emotionally and physically unbearable in the absence of anesthesia. Finally, OC cannot be used in prepubertal girls whose hypothalamic-pituitary axis not mature or ovulation does not occur.

\section{Ovarian tissue cryopreservation}

OTC seems to be advantageous as it can be performed at any time, with less delay to initiating cancer treatment. More than 60 cases of successful live births using tissue cryopreservation have been reported worldwide, however, it should still be considered experimental because there is no standard protocol or report with robust success rates.

Minimally invasive laparoscopic surgery is preferred and in this process, strips of ovarian cortical tissue, which contains vast amounts of primordial follicle, are harvested. The ovarian tissue should be evaluated for evidence of metastasis or malignant disease. In pediatric patients, it is preferred to freeze strips from the ovarian cortex than the whole ovary, the ovarian cortex is dissected into 1 - to 2-mm-thick strips after separation from medulla.

If ovarian insufficiency is diagnosed and the patient wants to be pregnant after complete cure of malignancy, orthotopic or heterotropic transplantation may be performed. Restoration of ovarian function was observed 3-6 months after reimplantation, documented blood level of several hormones changes - increased estradiol and decreased FSH levels. After ovarian tissue transplantation, additional assisted reproductive techniques may be often required. A recent meta-analysis has reported a CPRs of 57.5\% and a live birth rate of $37 \%$ after ovarian tissue transplantation. ${ }^{29)}$ 
However, there is a significant concern for oncologic reintroducing in patients with such as ovarian and hematological malignancies, as well as cancer with high risk of ovarian metastases and in fact, tissue cryopreservation and transplantation cannot be offered for most pediatric malignancies. The conflict among both the oncologists and the fertility specialists about the ovarian transplantation in cancer patients needs to be addressed in future research.

OTC is the only fertility preservation option for prepubertal females, but tissue from pre-pubertal girls contains only immature follicles. It's not an easy process to make immature follicles to be fully grown into mature and support the mature oocytes until in vitro fertilization. However there are 2 case reports of live births in patients who underwent tissue cryopreservation prior to menarche, indicating promising procedure in this population. $^{30,31)}$

\section{Ovarian suppression}

Ovarian suppression using gonadotropin-releasing hormone $(\mathrm{GnRH})$ analogues for during chemotherapy is one of the suggested fertility preservation method. It generates a pseudoprepubertal hormonal state and reduced utero-ovarian perfusion. In addition, possible hypotheses are suppression of FSH secretion, reduction of uterine-ovarian circulation, inhibition of GnRH receptor activation, and inhibition of upregulation of intragonadal antiapoptotic molecules. ${ }^{32)}$ Although a pseudoprepubertal hormonal state may make ovaries less vulnerable to gonadotoxic treatment, the protective effect of GnRH agonist during chemotherapy is under debate and still considered investigational. Oktay et al. ${ }^{33)}$, and Lambertini et al. ${ }^{34)}$ reported that ovarian suppression with GnRH agonists during chemotherapy reduce the risk of premature ovarian failure. Furthermore, in a recent meta-analysis, it is demonstrated that administration of GnRH agonists resulted in a higher rate of resuming menstruation and probability of pregnancy. ${ }^{35,36)} \mathrm{On}$ the contrary, Elgindy et al. ${ }^{37)}$ reported that the application of GnRH agonists during chemotherapy does not protect any of ovarian reserve parameters and not appear to protect the gonad from toxicity of chemoagents in other meta-analysis and systematic review. Although this treatment is still considered debatable by some authors, the use of GnRH agonists before and during chemotherapy is recommended by the authors of a recent Cochrane database analysis. ${ }^{38)}$ The above mentioned studies analyzed the adult (postpubertal) population and such treatments are not recommended for prepubertal girls who lack of maturity of the ovarian cycle.

\section{Conclusion}

Pediatric or adolescent patients with malignancy should be referred to a reproductive endocrinologist as soon as possible upon receiving a cancer diagnosis. Early referral will make patients and parents to consider an appropriate method of fertility preservation. Currently available options for fertility preservation for pediatric or adolescent patients with malignancy include cryopreservation of oocytes, and ovarian tissue before chemotherapy. Also GnRH analogues can be considered as another option during chemotherapy. An appropriate option of fertility preservation should be chosen through discussion between individual patients, their parents and healthcare providers with analyses of possible options.

\section{Conflict of interest}

No potential conflict of interest relevant to this article was reported.

\section{Acknowledgement}

This work was supported by the Ministry of Science, ICT and Future Planning (2016R1E1A1A01943455).

\section{References}

1 CureSearch. Childhood cancer statics [Internet]. Bethesda (MD): CureSearch; [cited 2018 Mar 1]. Available from: https://curesearch.org/Childhood-Cancer-Statistics.

2. Ministry of Health \& Welfare, National Cancer Center, Korea Central Cancer Registry. Annual report of cancer statistics in Korea in 2015, Goyang (Korea): National Cancer Center; 2017.

3. National Cancer Institute. Cancer in children and adolescents [Internet]. Bethesda (MD): National Cancer Institute; [cited 2018 Mar 1]. Available from: http://www. cancer.gov/types/childhood-cancers/child-adolescentcancers-fact-sheet.

4. Loren AW, Mangu PB, Beck LN, Brennan L, Magdalinski AJ, Partridge AH, et al. Fertility preservation for patients with cancer: American Society of Clinical Oncology clinical practice guideline update. J Clin Oncol 2013;31:2500-10.

5. Practice Committee of American Society for Reproductive Medicine. Fertility preservation in patients undergoing gonadotoxic therapy or gonadectomy: a committee opinion. Fertil Steril 2013;100:1214-23.

6. Adams E, Hill E, Watson E. Fertility preservation in cancer survivors: a national survey of oncologists current knowledge, practice and attitudes. Br J Cancer 2013;108:1602-15.

7. Forman EJ, Anders CK, Behera MA. A nationwide survey of oncologists regarding treatment-related infertility and fertility preservation in female cancer patients. Fertil Steril 2010;94:1652-6.

8. Yee S, Fuller-Thomson E, Lau A, Greenblatt EM. Fertility preservation practices among Ontario oncologists. J Cancer Educ 2012;27:362-8.

9. Ethics Committee of American Society for Reproductive Medicine. Fertility preservation and reproduction in 
patients facing gonadotoxic therapies: a committee opinion. Fertil Steril 2013;100:1224-31.

10. Lee S, Ozkavukcu S, Heytens E, Moy F, Oktay K. Value of early referral to fertility preservation in young women with breast cancer. J Clin Oncol 2010;28:4683-6.

11. Kim J, Mersereau JE. Early referral makes the decisionmaking about fertility preservation easier: a pilot survey study of young female cancer survivors. Support Care Cancer 2015;23:1663-7.

12. Chemaitilly W, Mertens AC, Mitby P, Whitton J, Stovall M, Yasui Y, et al. Acute ovarian failure in the childhood cancer survivor study. J Clin Endocrinol Metab 2006;91:1723-8.

13. Bedoschi G, Navarro PA, Oktay K. Chemotherapy-induced damage to ovary: mechanisms and clinical impact. Future Oncol 2016;12:2333-44.

14. Green DM, Nolan VG, Goodman PJ, Whitton JA, Srivastava $\mathrm{D}$, Leisenring WM, et al. The cyclophosphamide equivalent dose as an approach for quantifying alkylating agent exposure: a report from the Childhood Cancer Survivor Study. Pediatr Blood Cancer 2014;61:53-67.

15. Meirow D. Reproduction post-chemotherapy in young cancer patients. Mol Cell Endocrinol 2000;169:123-31.

16. Bakker B, Oostdijk W, Bresters D, Walenkamp MJ, Vossen JM, Wit JM. Disturbances of growth and endocrine function after busulphan-based conditioning for haematopoietic stem cell transplantation during infancy and childhood. Bone Marrow Transplant 2004;33:1049-56.

17. Wallace WH, Thomson AB, Kelsey TW. The radiosensitivity of the human oocyte. Hum Reprod 2003;18:117-21.

18. Rodriguez-Wallberg KA, Oktay K. Fertility preservation during cancer treatment: clinical guidelines. Cancer Manag Res 2014;6:105-17.

19. Wallace WH, Thomson AB, Saran F, Kelsey TW. Predicting age of ovarian failure after radiation to a field that includes the ovaries. Int J Radiat Oncol Biol Phys 2005;62:738-44.

20. Brougham MF, Wallace WH. Subfertility in children and young people treated for solid and haematological malignancies. Br J Haematol 2005;131:143-55.

21. De Bruin ML, Van Dulmen-den Broeder E, Van den Berg MH, Lambalk CB. Fertility in female childhood cancer survivors. Endocr Dev 2009;15:135-58.

22. Jadoul P, Dolmans MM, Donnez J. Fertility preservation in girls during childhood: is it feasible, efficient and safe and to whom should it be proposed? Hum Reprod Update 2010;16:617-30.

23. Salama M, Isachenko V, Isachenko E, Rahimi G, Mallmann P. Updates in preserving reproductive potential of prepubertal girls with cancer: systematic review. Crit Rev Oncol Hematol 2016;103:10-21.

24. Burns KC, Hoefgen H, Strine A, Dasgupta R. Fertility preservation options in pediatric and adolescent patients with cancer. Cancer 2018;124:1867-76.

25. Borini A, Lagalla C, Bonu MA, Bianchi V, Flamigni C, Coticchio G. Cumulative pregnancy rates resulting from the use of fresh and frozen oocytes: 7 years' experience. Reprod Biomed Online 2006;12:481-6.
26. Grifo JA, Noyes N. Delivery rate using cryopreserved oocytes is comparable to conventional in vitro fertilization using fresh oocytes: potential fertility preservation for female cancer patients. Fertil Steril 2010;93:391-6.

27. Kim TJ, Laufer LR, Hong SW. Vitrification of oocytes produces high pregnancy rates when carried out in fertile women. Fertil Steril 2010;93:467-74.

28. Noyes N, Porcu E, Borini A. Over 900 oocyte cryopreservation babies born with no apparent increase in congenital anomalies. Reprod Biomed Online 2009; 18:769-76.

29. Pacheco F, Oktay K. Current success and efficiency of autologous ovarian transplantation: a meta-analysis. Reprod Sci 2017;24:1111-20.

30. Demeestere I, Simon P, Dedeken L, Moffa F, Tsépélidis $\mathrm{S}$, Brachet $\mathrm{C}$, et al. Live birth after autograft of ovarian tissue cryopreserved during childhood. Hum Reprod 2015;30:2107-9.

31. Walsh F. Woman has baby using ovary frozen in childhood. BBC News [Internet]. 2016 Dec 14 [cited 2018 Mar 1]; Health [about 3 p]. Available from: http://www.bbc.com/ news/health-38312995.

32. Blumenfeld $Z$. How to preserve fertility in young women exposed to chemotherapy? The role of GnRH agonist cotreatment in addition to cryopreservation of embrya, oocytes, or ovaries. Oncologist 2007;12:1044-54.

33. Oktay K, Rodriguez-Wallberg K, Munster P. Ovarian protection during adjuvant chemotherapy. N Engl J Med 2015;372:2268-9.

34. Lambertini M, Boni L, Michelotti A, Gamucci T, Scotto T, Gori S, et al. Ovarian suppression with triptorelin during adjuvant breast cancer chemotherapy and long-term ovarian function, pregnancies, and disease-free survival: a randomized clinical trial. JAMA 2015;314:2632-40.

35. Del Mastro L, Ceppi M, Poggio F, Bighin C, Peccatori F, Demeestere I, et al. Gonadotropin-releasing hormone analogues for the prevention of chemotherapy-induced premature ovarian failure in cancer women: systematic review and meta-analysis of randomized trials. Cancer Treat Rev 2014;40:675-83.

36. Munhoz RR, Pereira AA, Sasse AD, Hoff PM, Traina TA, Hudis CA, et al. Gonadotropin-releasing hormone agonists for ovarian function preservation in premenopausal women undergoing chemotherapy for early-stage breast cancer: a systematic review and meta-analysis. JAMA Oncol 2016;2:65-73.

37. Elgindy E, Sibai H, Abdelghani A, Mostafa M. Protecting ovaries during chemotherapy through gonad suppression: a systematic review and meta-analysis. Obstet Gynecol 2015;126:187-95.

38. Chen H, Li J, Cui T, Hu L. Adjuvant gonadotropin-releasing hormone analogues for the prevention of chemotherapy induced premature ovarian failure in premenopausal women. Cochrane Database Syst Rev 2011;(11):CD008018.

39. Winkler-Crepaz K, Ayuandari S, Ziehr SC, Hofer S, Wildt L. Fertility preservation in cancer survivors. Minerva Endocrinol 2015;40:105-18. 